\title{
MORTALITY OF FISH FRY AS A RESULT OF SPECIFIC AND ASPECIFIC CERCARIAL INVASION UNDER EXPERIMENTAL CONDITIONS
}

\author{
G. MAJOROS* \\ Central Veterinary Institute, H-1149 Budapest, Tábornok u. 2, Hungary \\ (Received February 8, 1999; accepted April 8, 1999)
}

\begin{abstract}
Although the effect exerted on fish by fluke cercariae developing in fish as intermediate hosts is fairly well known, cercariae of flukes requiring intermediate hosts other than fish occur in fish ponds in much higher numbers. To study the effect of these latter cercariae on fish fry, a few days old common carp fry (Cyprinus carpio) were exposed to cohorts of xiphidiocercariae or echinocercariae shed by one or two snails acting as intermediate hosts. The observed pathogenicity of these cercariae was compared to the well-known pathogenic effect of Diplostomum cercariae. Although Diplostomum cercariae proved to be the most pathogenic, occasionally also the other two types of cercariae could kill the common carp fry by invading their body. Xiphidiocercariae ingested by the fish penetrated the intestinal wall and developed into metacercariae within the body of the fry. They could also get into the musculature through the skin. The much larger echinocercariae invaded the gill cavity where they became encapsulated, thus hampering gill function and leading to fish mortality due to oxygen deficiency. Diplostomum cercariae were more pathogenic than the cercariae of the other two flukes in that they were shed by the intermediate host in very large numbers. Although much less xiphidiocercariae and echinocercariae emerged from the intermediate host snails than Diplostomum cercariae, the former could kill the fish fry already in lower numbers. These findings prove that fluke cercariae nonspecific to fish may exert pathogenic effects on fish, and presumably contribute to the early mortality of young fry placed out into fish ponds.
\end{abstract}

Key words: Cercaria, Diplostomum sp., xiphidiocercaria, echinocercaria, experimental cercarial invasion, pathogenic effect, fish fry

The natural mortality rate of young fish fry placed out into fish ponds and the causes of their mortality are difficult to study, as dead fish a few centimetres in size disappear on the bottom and become inaccessible for pathological examinations. At the same time, fish breeders not infrequently find that in certain rearing ponds young fry disappear soon after having been placed out, almost

*E-mail: majoros@ccmail.oai.hu; Fax: +36 (1) 2525177 
overnight. This early mortality is always limited to certain ponds, and in the affected ponds almost the entire stock of fish fry placed out at a time will be lost. This is what happened in the summer of 1998 in a fish farm situated in the Great Hungarian Plain where all fish died in a rearing pond next to the fish hatchery. After letting the water out of the pond, enormous masses of lymnaeid pond snails, acting as an intermediate hosts in the development of trematodes, were seen to cover the bottom of the lake in numbers reaching several thousand snails per square metre (Gy. Csaba, personal communication).

Certain authors explain that phenomenon by the damage done by the glochidia of mussels (Dobó, 1994), while others attribute that mortality, typically occurring in the spring or early summer, to cercarial invasion. Both practical experience (Kocylowski and Miaczynski, 1963) and laboratory investigations (Sommerville, 1982) have clearly demonstrated that the cercariae of fish-specific trematodes can cause mortality in young fish fry. In addition, empirical data indicate that fish fry can be killed by the cercariae of any trematode species that are capable of invading them (Roberts, 1989). Invasion by trematode larvae causing mortality or pathological emaciation of fish has been reported by Palmer (1939; cit. by Inchausty et al., 1997), Bauer et al. (1964), Ishkov and Batchinskii (1969), Hoffmann et al. (1990) and Ballabeni (1994). In Hungary only a single, well-documented study has been published on that pathological process, which reported mortality caused by Diplostomum larvae in 3-week-old grasscarp fry reared in a fish farm (Molnár, 1974).

The cercariae of numerous trematode species develop in European freshwater molluscs, but only a small proportion of them reach maturity in fishes (Zdárská, 1963; Ginetsinskaya, 1988). Not only obligate fish-parasitic trematode larvae occur in the fish ponds either. In most periods of the year, mainly cercariae of flukes specific for frogs, snakes, small mammals and aquatic birds can be found in the ponds of Hungarian fish farms (Majoros, 1996).

In a preliminary survey the present author (Majoros, 1997) already studied the ability of cercariae isolated from snails to invade and develop in young fry and several months old common carp. He has found that in certain cases very young fish fry can be killed by the cercariae of flukes incapable of infecting older fish, which, on the basis of their morphological features, were identified as trematodes specific for hosts other than fish.

In the experiments presented here, the pathogenicity of the cercariae of two trematode species belonging to two different trematode families and incapable of further development in fish was studied in common carp fry up to about one $\mathrm{cm}$ in size and reared in an infection-free environment. It was compared with the effects of larvae of the strictly fish-specific Diplostomum sp. known to be pathogenic to fish. 


\section{Materials and methods}

Common carp (Cyprinus carpio) fry aged 4-20 days, hatched in Zuger jars from artificially stripped eggs and reared in a hatchery in parasite-free environment were used as experimental animals. The fry were kept in 10-litre aquaria with flow-through water until the start of the infection experiment, and fed on ground boiled eggs and then on dry, artificial eel food made from meat meal with a particle size of 1-2 mm. Before the start of the infection experiment the fry, in groups of four or five, were placed into glass jars each containing $500 \mathrm{ml}$ stale tap-water.

The snails were collected from different fish ponds (Lőrinci, Százhalombatta, Hortobágy) and from natural lakes (Lipót). In the laboratory, the molluscs were placed, one by one, into $200 \mathrm{~cm}^{3}$ glass dishes containing dechlorinated tapwater, and the dishes were illuminated with yellowish-white neon light (F29 Warm White) for at least half a day during the daytime. If subsequently, or after keeping them in the dark overnight, cercariae emerged from the snails, the cercariae were examined, and the snail specimens shedding cercariae of identical morphology were grouped. Only the infected snails were used for the further experiments. The snails were kept in the same room as the fish, under the same illumination.

Cercariae that had newly emerged from the snails were dropped into eggwhite and examined alive with a light microscope, using differential interference contrast technique. To take their measurements, cercariae were fixed with cold formalin under a coverslip.

During the infection experiments, 4-5 fish fry and one or two snails shedding cercariae were placed into $500 \mathrm{ml}$ dechlorinated, oxygen-rich, stale tapwater. If earlier the shedding of cercariae was found to be triggered by illumination (Diplostomum furcocercariae, echinocercariae), the experimental glass was exposed to illumination in a similar manner as at the time of selecting the infected snails. If cercarial shedding showed a diurnal pattern independent of light (xiphidiocercariae), we waited till emergence occurred. The fish were placed into the experimental dishes directly after the emergence of cercariae, and the snail was also left beside the fish so that the latter could always meet newly emerged cercariae. There was no aerator in the 0.5-litre glass dishes, as its operation would have affected the observation and the movement of cercariae. Concurrently with the fish to be infected, in separate vessels groups of five fish were kept under similar conditions but without a snail, for monitoring the development of a possible oxygen deficiency.

The number of cercariae present in the water of the experimental fish was estimated by counting formalin-killed cercariae present in $5 \mathrm{ml}$ of water removed from the dish with a pipette.

The fish exposed to cercarial invasion were inspected at 10- to 20-min intervals for at least $6 \mathrm{~h}$. If they did not die during that period, they were left beside 
the infected snails till the subsequent day. The live fish spent at least $24-26 \mathrm{~h}$ in the infection jar together with the snails and the cercariae. Further on only those fish were studied in detail which had died during the above period. If the fish showed abnormal behaviour before death, thus indicating that infection had occurred, they were removed from the water immediately after they had ceased to move. Death was considered to have occurred when excitability and spontaneous movement ceased. The dead fish were fixed in $10 \%$ buffered formalin $(\mathrm{pH} \mathrm{7.0)}$, embedded in paraffin, and $10 \mu \mathrm{m}$ thick longitudinal serial sections were made from their entire body and stained with haematoxylin and eosin.

In the case of fish infected with xiphidiocercariae, after fixation the entire bodies of three fish each were steeped in haemalum solution. After removing the excess staining solution with lactic acid, the fish were rendered transparent with the $50 \%$ solution of Macrogol-400 polyoxaethene. As a result, the cercariae having penetrated into them and the metacercariae developing from the cercariae became well distinguishable from the surrounding tissues (Majoros, unpublished).

For the visual observation of cercarial penetration we chose echinocercariae as we could not find data on their penetration into vertebrates, and also because they were the largest of the cercariae studied. Under the experimental conditions described above, we placed 100-150 of the collected cercariae beside 5 young guppies (Poecilia reticulata) measuring $25 \mathrm{~mm}$, and we observed the movement of the fish and the cercariae which were just visible with the unaided eye. During the infection experiment we placed a few drops of $1 \%$ neutral red solution into the 0.51 of water. That amount of dye stained the live cercariae red within a few minutes, thus making their movement even easier to follow. The fish were observed for $5 \mathrm{~h}$, then exterminated by cervical dislocation.

\section{Results}

\section{Cercariae used for the infection}

Diplostomum furcocercaria (Fig. 1). These cercariae emerged from Lymnaea auricularia snails originating from Százhalombatta, especially after intense illumination in the morning. As many as 6000-8000 cercariae emerged from an adult snail of $3 \mathrm{~cm}$ shell height a day.

Rapidly moving cercaria with bifurcated tail. Resting position with tail stem bent at an angle somewhat less than $90^{\circ}$, furcae open at an obtuse angle (Fig. 1a). Cercarial body (Fig. 1b) measuring 220-250 ×50-60 $\mu \mathrm{m}$. Acetabulum situated at the border of the aboral third, its diameter is nearly equal with that of the anterior organ. In the body, 6 flame cells and at least two pairs of ciliary tufts can be observed on both sides. Excretory vesicle simple, non-lobate. Four unicellular posta- 
cetabular penetration glands opening in the oral part lie side by side. Prepharynx slightly dilates before the muscular pharynx. Intestinal bifurcation above the acetabulum, caeca extend to the excretory vesicle. Aggregations of yellowish pigment granules at the intestinal bifurcation. Body spination is shown in Fig. 1c.

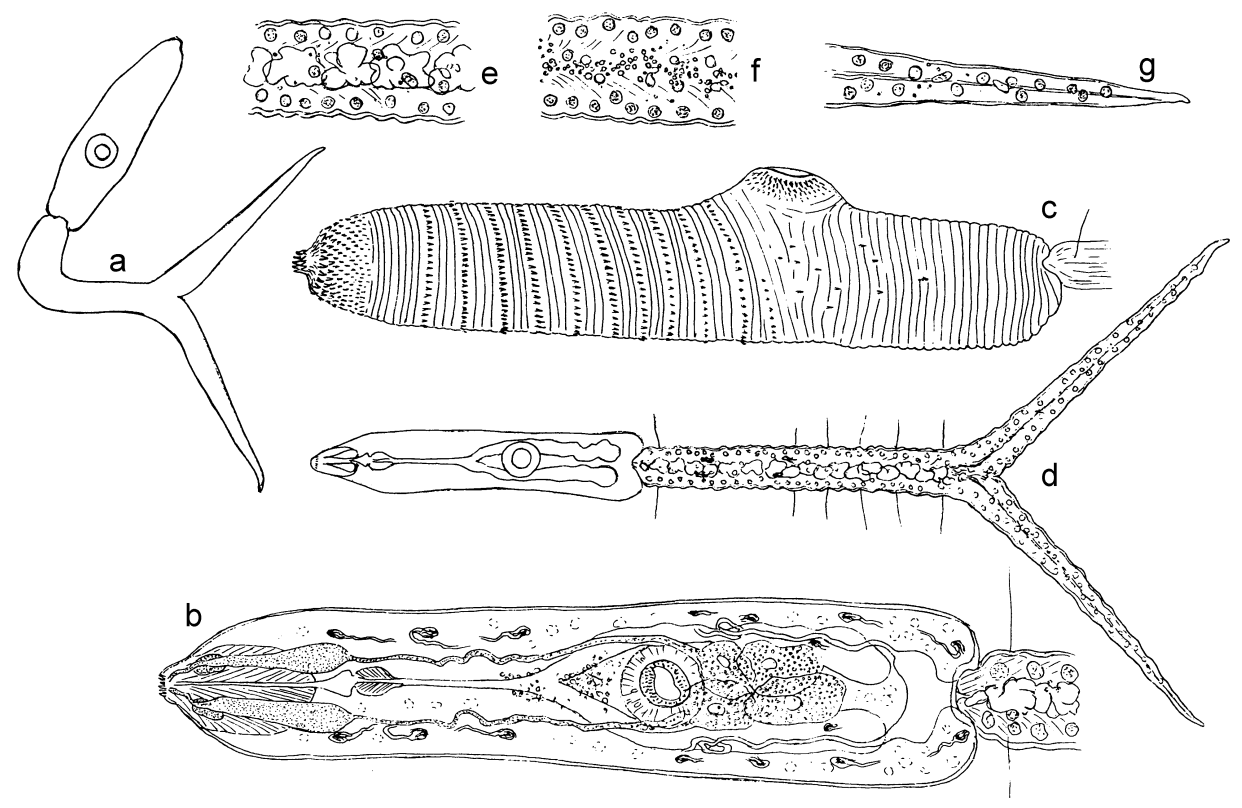

Fig. 1. Furcocercaria of Diplostomum sp. from a Lymnaea auricularia snail (Százhalombatta, Warm-water Fish Hatchery). (a) live cercaria in resting position; (b) cercarial body; (c) surface of the cercarial body; (d) whole cercaria in fixed condition; (e) caudal bodies in the median line of the tail, immediately after emergence; (f) remnants of caudal bodies 6-7 h after emergence; (g) tail end with the terminal part of the central bundle

Tail stem and furcae of formalin-killed larvae are as long as the body (Fig. 1d). Tail stem contains two pairs of flame cells and 15-16 caudal bodies. Caudal bodies have irregularly lobate surface immediately after larval emergence (Fig. 1e) and become resorbed within 5-6 h or at most half a day thereafter, leaving behind a granular material (Fig. 1f). Furcae contain no caudal bodies, and the fibre bundle running in their axis extends almost to the tail tip (Fig. 1g). On the tail stem there are at least six pairs of bristles, one pair at the tail stem while the other pairs separated from and caudal to the former (Fig. 1d).

Xiphidiocercaria (Fig. 2). These cercariae emerged from L. auricularia snails originating from Hortobágy and Lörinci, in the early morning or in the evening hours, irrespective of illumination. Only 150-200 larvae emerged from a snail of $2-3 \mathrm{~cm}$ shell height at a time. 


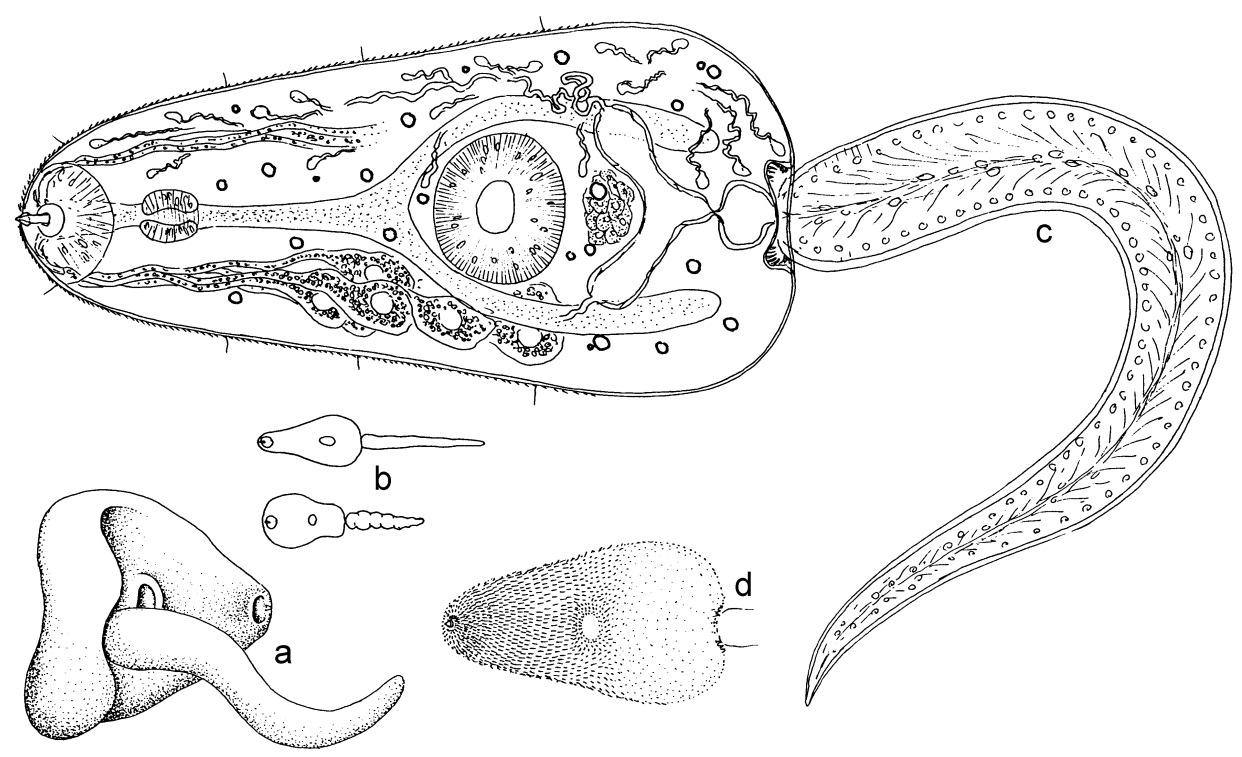

Fig. 2. Xiphidiocercaria from a Lymnaea auricularia snail (Hortobágy, Gyökérkút fish ponds). (a) live cercaria in resting position; (b) two characteristic phases of the movement of cercaria crawling on the bottom; (c) whole cercaria, with the penetration glands indicated on the left and flame cells on the right; (d) pattern of spination of the body surface

A cercaria of medium size and not too fast movement, swimming with vibrating movement in oral direction. In resting position its body is bent in ventral direction, the tail is floating atonically, and the larva often sinks to the bottom of the water with its dorsal part downwards (Fig. 2a). It crawls forward on its ventral side with vermicular movement that consists of longitudinal waves occurring at intervals of 2-3 seconds, narrowing or widening according to the muscle contractions (Fig. 2b). Sometimes, mainly when changing direction, the body makes spanning movements.

Cercarial body (Fig. 2c) measuring 280-310 × 100-130 $\mu \mathrm{m}$. Acetabulum located directly behind the transverse mid-length of body, $50-60 \mu \mathrm{m}$ in diameter, slightly larger than the oral sucker. Stylet located at the anterior end, 10$15 \mu \mathrm{m}$ long, with a slightly widened base. Body contains numerous highly refractive lipid droplets. Excretory ducts are barely visible and there are at least 18 pairs of flame cells. Prepharynx short, caeca dividing above the acetabulum reach to the terminal chamber of the three-chamber excretory vesicle. Penetration glands extend from the anterior end to the aboral margin of the acetabulum, and there are 6 glandular cells on both sides. The cell group of the genital primordium is located at the concave surface of the proximal chambers of the ex- 
cretory vesicle. Tail non-bifurcated, without fin-fold, somewhat longer than body $(350-400 \mu \mathrm{m})$, and contains no excretory collecting canals.

Body surface covered with spines that grow smaller backwards from the anterior end (Fig. 2d). Larger spines can be seen in the hollow surrounding the tail stem. There are at least 4 sensory hairs on both sides of the body.

Echinocercaria (Fig. 3). This cercaria was obtained from Lymnaea stagnalis snails originating from Lipót and Lörinci. It emerged mainly on the effect of intense illumination in the morning. Although the intermediate host snails of 5-6 cm shell length were much larger than L. auricularia, only 50-150 cercariae emerged from them at a time.

Cercaria of slow movement and large body size; by moving its tail it barely moves forward; rather, it floats to and from in the water. It does not have a characteristic resting position; with body and tail stretched out on the bottom and using its suckers for adhesion, it crawls forward on its ventral side with spanning and longitudinal movement.

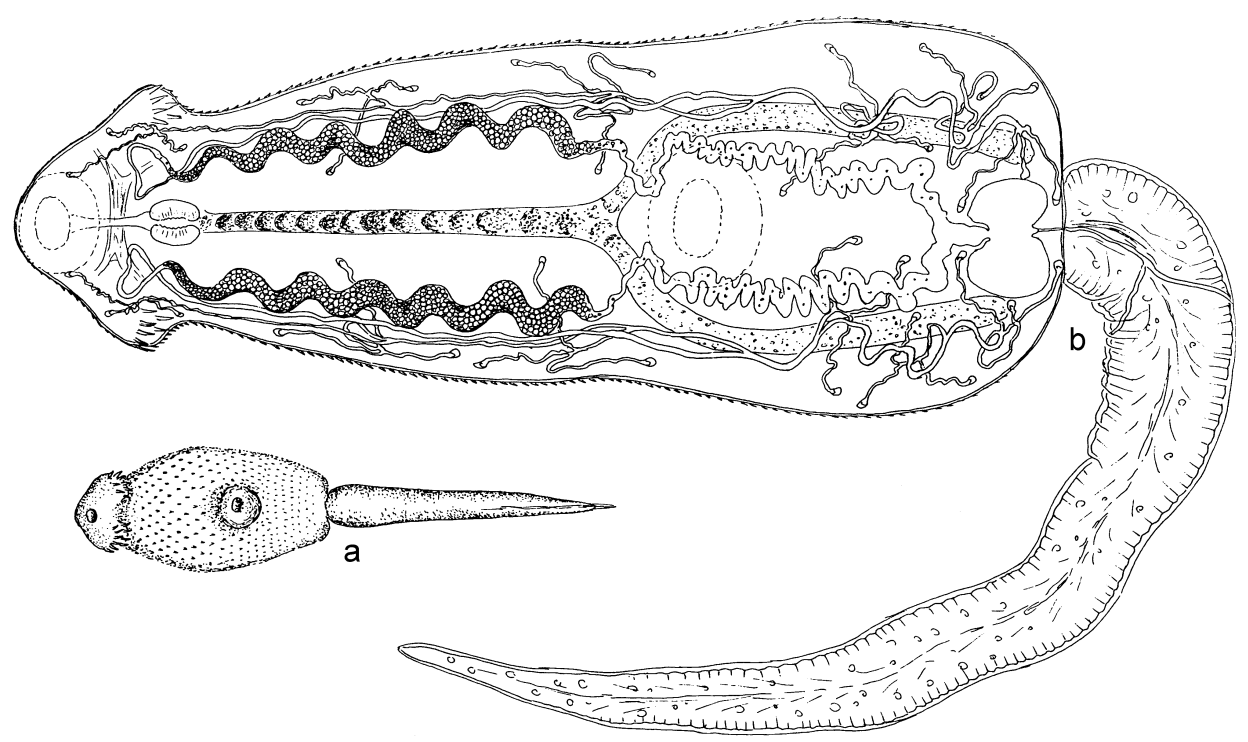

Fig. 3. Echinocercaria from a Lymnaea stagnalis snail (Lipót, Holt-Duna). (a) live, resting cercaria, with pattern of spination of the body surface; (b) whole cercaria, with the intestine and ducts of the excretory system shown in the cercarial body

The formalin-fixed cercarial body measures 350-400 × 140-150 $\mu \mathrm{m}$ (Fig. $3 b)$. Oral sucker is surrounded by 35-40 spines at the edge of the aboral disk. Acetabulum slightly wider than oral sucker and lies beneath the transverse midlength of body. Prepharynx short, intestine runs at length from the muscular 
pharynx to the acetabulum where it bifurcates. Caeca reach the middle of excretory vesicle. Especially in oral direction, intestine is filled with granular material aggregated into bolus-like masses. Flame cell formula $2[(3+3+3)+(3+$ $3+3)]=36$. The dilated, oral portion of the descending duct of the excretory system is filled with numerous, more than one hundred granules.

Tail 450-500 $\mu \mathrm{m}$ long in elongated condition. Terminal duct of excretory system leaving the excretory vesicle ends in a bifurcation in the oral part of the tail. Starting from the terminal third, the tail end is bordered by a very narrow dorsoventral fin-fold.

Body surface covered with scale-like, flat spines arranged diagonally in rows posterior to the margin of the adoral disk. These spines grow smaller posteriorly and disappear near the tail (Fig. 3a). There are no tegumental spines around the oral sucker. By light microscopic techniques no sensory hairs can be seen on the cercarial body.

\section{Fish infection experiments}

(A) Effect and location of Diplostomum cercariae. As many as 100-200 cercariae required $1-3 \mathrm{~h}$ to kill the five $1 \mathrm{~cm}$ long common carp fry placed into each of the three dishes containing 0.5 litre of water each. Two to three minutes after the cercariae had been placed in, the fish became restless and started to dart in the water in a zigzag line with a jerky progressive movement. A further few minutes later the intensive swimming was replaced by periods of rest. At such times the fish tilted on their side and started to sink, then tried to swim again, but they did not come up to the surface of the water and were not gasping for air. They did not die simultaneously; rather, their active movement ceased in one or two hours, and finally all the fish were lying motionless on the bottom of the dish. At that time they usually still responded to mechanical stimuli and their operculum was moving at a slow pace. Complete immobility set in about half an hour or one hour thereafter. The fish died with their operculum open or closed.

At the same time when the movement of fish became abnormal, the upper part of the head, especially the crown of head above the brain, became reddened. The other parts of the body turned pale, but the visibility of that markedly depended on individual variation in the pigment content of the integument.

Percutaneously penetrated cercariae having lost their tail could be observed in the body of fish already in unstained condition. The white flukes crawling on the body surface and in the epithelium could be seen by stereomicroscopy; in unstained condition they were especially well visible in the transparent fins devoid of pigment (Fig. 4a). 

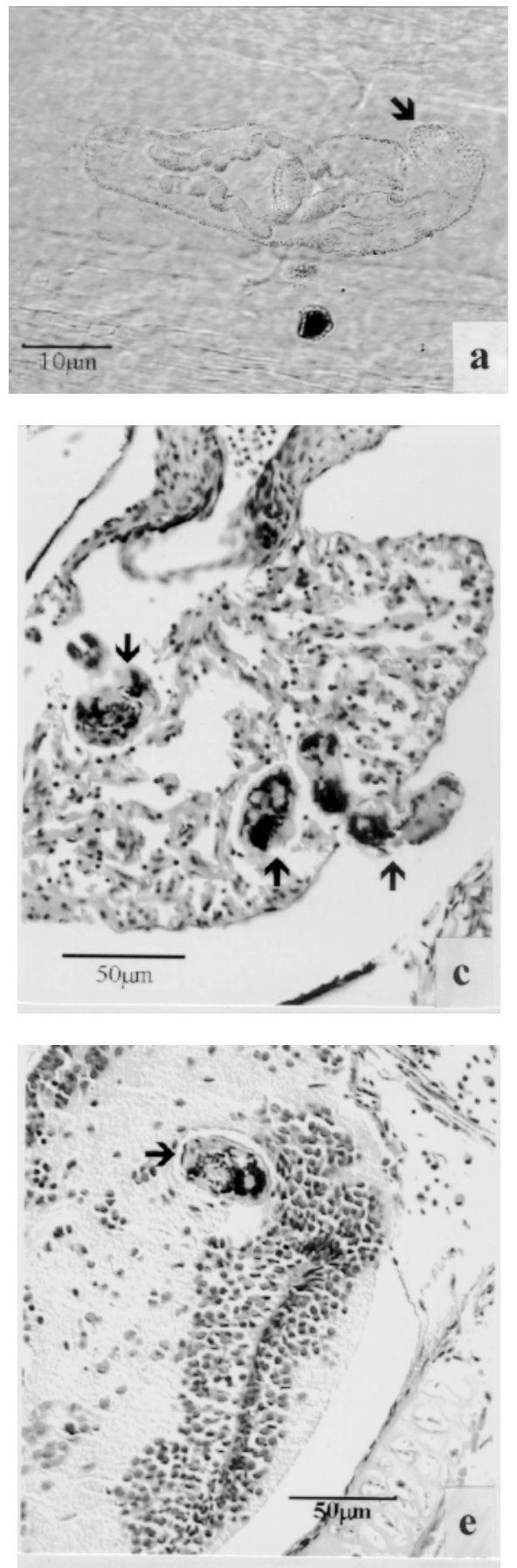
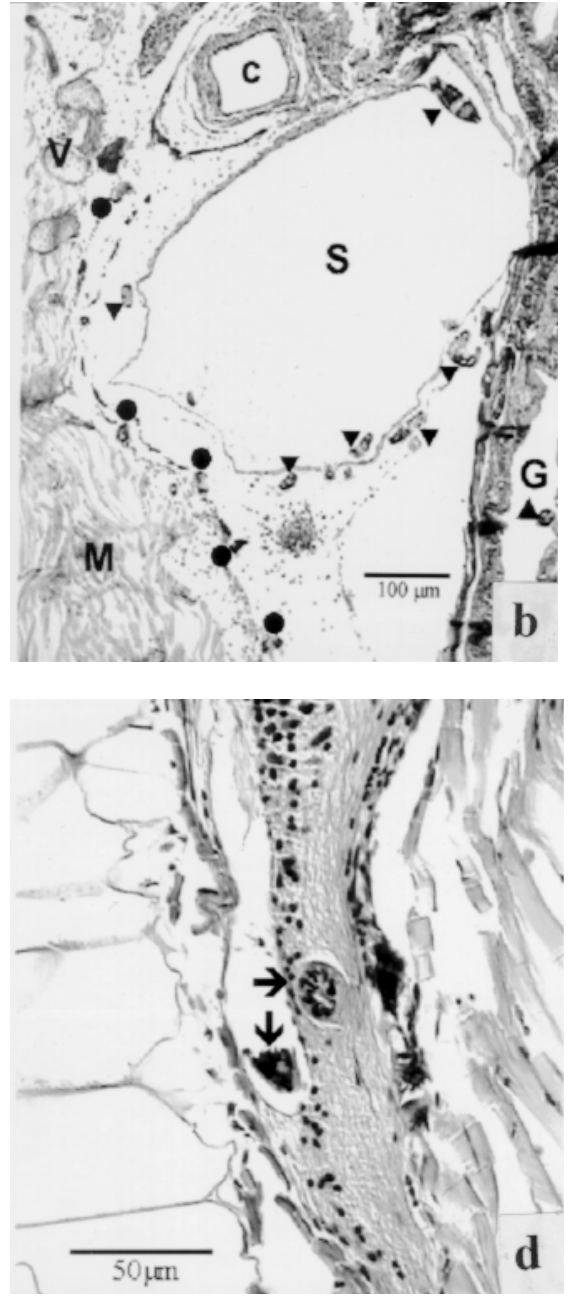

Fig. 4. Diplostomum metacercariae in common carp fry. (a) Diplostomum larva marked with an arrow at the oral end of the body, located near the cartilaginous fin ray of the caudal fin (fresh preparation). Sections stained with haematoxylin and eosin: (b) Larvae around the swimbladder $(\boldsymbol{\nabla})$, along the serous membrane $(\mathbf{O})$ and in the gut $(\mathbf{A})$. $\mathrm{S}=$ swimbladder; $\mathrm{G}=$ gut; $\mathrm{M}=$ musculature of the trunk; $\mathrm{V}=$ thoracic vertebrae;

$\mathrm{C}=$ crop. (c) Larvae in the wall of the heart ventricle $(\rightarrow)$. (d) Larvae in the spinal cord $(\rightarrow)$. (e) Larva in the brain $(\rightarrow)$ 
In histological sections, the invading parasites could be seen in almost all parts of the body; at the same time, they could not yet be detected in their final location, in the eye lens. The larvae, which measured $40-50 \mu \mathrm{m}$, were migrating primarily in the loose tissues and in the cavity systems among the parenchymal organs, thus in the abdominal cavity along the serous membranes (Fig. 4b), in the spinal canal and between the muscles. They also occurred in the gill, heart (Fig. 4c), spinal cord (Fig. 4d), and even in the brain (Fig. 4e).

No inflammatory reaction could be seen around the invading larvae, nor was their migration route marked by histologically detectable inflammatory cells. No local accumulation of the larvae was seen in any organ or body region; rather, the larvae were more or less uniformly distributed within the fish body.

(B) Effect and location of xiphidiocercariae. The xiphidiocercariae that emerged from snails (about 200-400 cercariae/litre of water) required a much longer time to kill the fish than did Diplostomum cercariae. Ten out of the 15 one-centimetre-long common carp fry placed into the 3 vessels containing 0.51 water each died within 6-22 h of exposure.

Large individual variation could be observed in the length of time that elapsed until death. Fish exposed to cercarial invasion began to show abnormal behaviour already within a few hours, and four fish died 6-7 h after exposure. Three fish died 20-21 h after challenge, while further three at the end of the 26-h period of observation. The remaining 5 fish did not die even $26 \mathrm{~h}$ postchallenge; however, by that time no live cercariae had remained in the water, nor did new cercariae emerge from the snails present in the dish.

From the beginning, all experimental fish tried to snap up the cercariae wriggling in the water, which were just visible with the unaided eye under direct illumination against a dark background. Of the fish which were placed among xiphidiocercariae and died, the behaviour of only those seven specimens could be observed immediately before death which died during the daytime. These fish did not display abnormally active movements; on the contrary, they were much calmer than the others. They consumed the larvae floating in the water like plankton in the same way as the fish that survived. Death occurred without any special premonitory signs: the fish were floating in the water in the same place, then turned on their side and sank to the bottom of the dish. Their body did not become discoloured, nor did they attempt to come up to the water surface. For some minutes, fish that had sunk to the bottom tried to rise and swim, but finally they remained on the bottom once and for all. They died with their operculum open, and excitability ceased almost immediately after the last attempt to swim. 
In the body of the dead fish as well as on the surface of their fins numerous greyish-white nodules could be seen. Each of these nodules contained a tailless, moving cercaria. In histological sections, some cercariae were seen 'naked' in the tissues, while others had formed a capsule around themselves (Fig. 5b). The cercariae assembled mainly in the wider anterior two-thirds of the body, with lower numbers present in the other parts. In fish rendered transparent it could be seen that cercariae had reached almost all parts of the body, and their total amount in the fish was not negligible even as compared to the host's own body weight (Fig. 5a).

Neither inflammatory reaction nor haemorrhage could be observed around the larvae that had penetrated into the body. Larvae occurred in the gut lumen itself, between the gut and the abdominal organs (Fig. 5c), among the gill lamellae, but mainly in the muscles under the skin (Fig. 5d). They did not penetrate into the central nervous system but got close to it in the muscles along the spine and on the surface of the skull.

Metacercariae starting to encapsulate were also seen attached to the body surface of surviving fish not showing abnormal signs, 5-12 metacercariae per fish. These fish were killed on the third day of the experiment. At that time, only barely recognisable remnants of cercariae could be found in their gut. There were no cercariae among the viscera.

(C) Effect and location of echinocercariae. At the start of the experiment, echinocercariae shed by L. stagnalis snails were used for infecting 15 one-centimetre-long common carp fry at a density as low as 40-50 cercariae/ 0.51 of water, since these cercariae were much larger than those of the trematode species discussed above, and emerged from the snails in much lower numbers at a time than the other cercariae. At the same time, during the daytime (in the hours of illumination) new cercariae kept emerging almost continuously from the snails placed beside the fish. Therefore, during the entire period of experiment the fish could be exposed to a similar number of cercariae as in the previous two experiments.

The echinocercariae, which were well visible to the naked eye, remained active in the water only for 3-4 h after emergence. Subsequently they were staying close to the bottom of the dish, showed little swimming activity and were spanning on the glass surface with characteristic vermicular movement. The small fish were apparently trying to snap them up. By the day after the start of the experiment, the cercariae had killed 6 out of the 15 fish. Only two fish could be observed for ante-mortem behaviour, as the other four died during the night. The two observed fish died $25 \mathrm{~h}$ after having been exposed to the cercariae. The onset of cercarial effect could not be determined, as cercariae were continuously present in the water. Fish showing signs were initially swimming about and gasping near the water surface, then their movement became slower, they sank to the bottom of the dish with their operculum open, and became motionless. 
It was also observed that these cercariae of large body size often adhered to the body surface of fish, although did not stay there for a long time. On each of the four fish that died at night there were 5-6 encapsulated echinocercariae; however, this may be considered a postmortem phenomenon, as encapsulated cercariae were consistently found also on other materials (fish and snail excrement) that had sunk to the bottom of the dish.
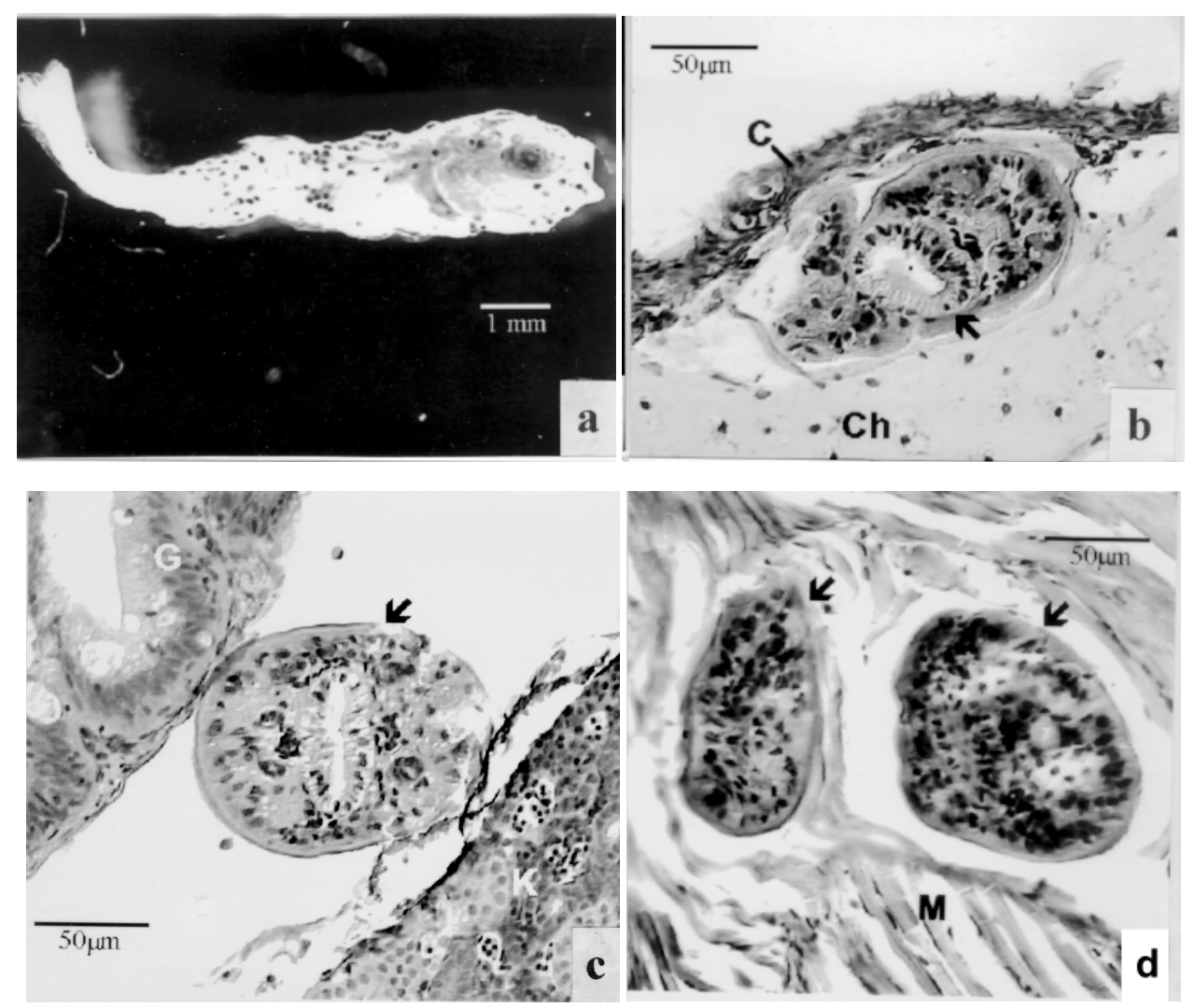

Fig. 5. Xiphidiocercariae in common carp fry. (a) Xiphidiocercariae having penetrated into the body of fry appear as dark spots in the preparation stained with haemalum and rendered transparent with polyoxaethene. Sections stained with haematoxylin and eosin: (b) Encapsulated xiphidiocercaria on the surface of the cranial chondroid tissue (Ch) of common carp fry, under the cutis (C).

The acetabulum is marked with an arrow $(\rightarrow)$. (c) Cross-section of a nonencapsulated xiphidiocercaria $(\rightarrow)$ in the body cavity between the kidney $(\mathrm{K})$ and the gut wall $(\mathrm{G})$.

(d) Nonencapsulated xiphidiocercariae in the body musculature (M)

Subsequent dissection of the Lymnaea snails serving as donor revealed that a certain proportion of the cercariae had returned into the snails and encapsulated in the tissues of their mantle cavity. 
Histological examination of the succumbed fish proved that they had eaten a large number of cercariae. The same was found when dissecting fish that had survived the challenge. The gut contained a large amount of well-discernible cercarial remnants (Fig. 6a), but the absence of larvae from the visceral organs indicated that the cercariae had been unable to penetrate the gut wall. At the same time, the gill cavity of succumbed fish always contained encapsulated, intact cercariae (Fig. 6b), at least three or four per fish. No tissue reaction was seen around them, at most the gill epithelium was missing around the wall of the capsule.
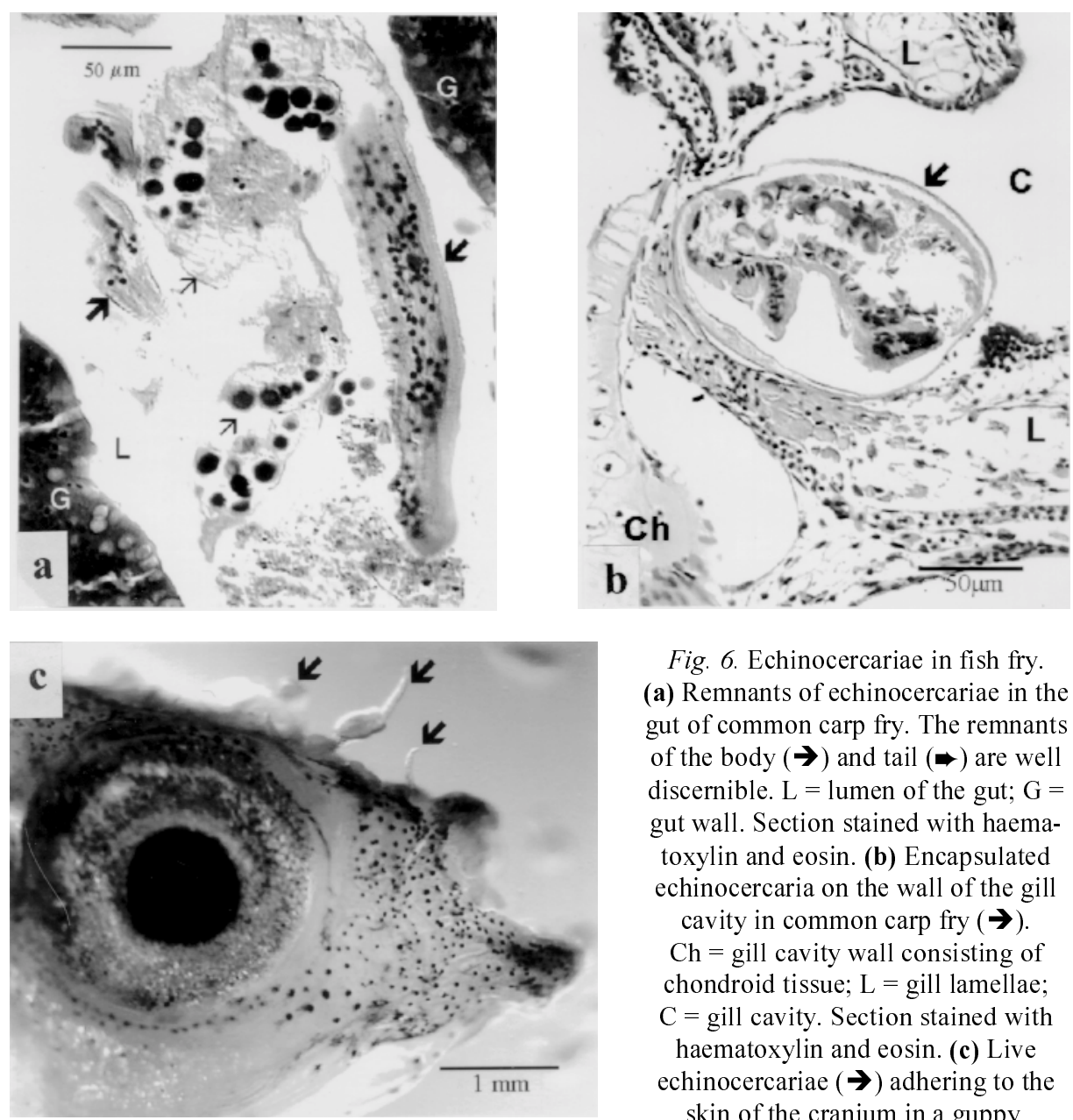

Fig. 6. Echinocercariae in fish fry. (a) Remnants of echinocercariae in the gut of common carp fry. The remnants of the body $(\rightarrow)$ and tail $(\rightarrow)$ are well discernible. $\mathrm{L}=$ lumen of the gut; $\mathrm{G}=$ gut wall. Section stained with haematoxylin and eosin. (b) Encapsulated echinocercaria on the wall of the gill cavity in common carp fry $(\rightarrow)$. $\mathrm{Ch}=$ gill cavity wall consisting of chondroid tissue; $\mathrm{L}=$ gill lamellae; $\mathrm{C}=$ gill cavity. Section stained with haematoxylin and eosin. (c) Live echinocercariae $(\rightarrow)$ adhering to the skin of the cranium in a guppy. Stereomicroscopic picture 
The mode of contact between the echinocercariae and the fish could be followed easily by placing beside the guppies cercariae that had been stained red with a vital dye. The cercariae did not follow the fish swimming in the water; rather, they were swimming about irregularly. The fish, on the other hand, snapped up the cercariae, but only the ones swimming in the water, and not those which were spanning on the wall of the dish. When, however, the cercariae came into contact with the body surface of the fish, they tried to adhere to it and move on it with a spanning movement (Fig. 6c). During their movement, the fish brushed off some of the cercariae crawling on them, but 10-15 cercariae always remained on each fish. Some of the cercariae shrank and became rounded off, i.e. started to encapsulate on the skin of the fish, but only superficially.

The gut of killed fish contained numerous destroyed cercariae. Two fish had one cercaria each stuck in the gill opening, but no cercariae were seen in the gill cavity or penetrated into the skin. Cercariae having started to encapsulate were observed on the skin. No cercariae were seen among the viscera and in the muscles.

\section{Discussion}

\section{Cercariae used for infection}

The Diplostomum furcocercaria used in the experiment most closely resembles the larvae of Diplostomum spathaceum. Based upon the description of Dubois (1970) and Yamaguti (1975) it somewhat differs from the latter in the distribution of bristles, in the number of the caudal bodies and in the pattern of the superficial spine rows; however, these traits do not necessarily prove that this furcocercaria belongs to some other species. D. spathaceum is a fairly common intestinal parasite species occurring in Hungary (Edelényi, 1974), which has been reported from several species of aquatic birds.

By its morphological structure, the xiphidiocercaria used in the present experiments could be classified into the Plagiorchioidea superfamily of Digenea, more closely into the Plagiorchiidae family (Schell, 1970, 1985). More precise classification was not possible on a morphological basis; however, judging from its structure and from data available on the occurrence of Hungarian trematodes it is very likely to have been the larval form of the fluke Ophisthyoglyphe ranae, which is widespread in the frog species of the genus Rana all over Europe (Edelényi, 1974) and commonly occurs in fish ponds, thus also in Hortobágy.

In its morphological features the echinocercaria studied by us is closest to the cercariae of the trematode species Echinostoma revolutum, which is a fairly common fluke parasitising the intestine of mallard (Vojtek, 1981), and is not rare in Hungary either (Edelényi, 1974). As the descriptions of cercariae belonging to 
the Echinostomatidae family and having a known developmental cycle are inconsistent (Yamaguti, 1975; Niewiadomska et al., 1997), the species of the larva found by us could not be conclusively determined without an infection experiment.

\section{Infection experiments in fish}

The results of the present experiments allow us to conclude that young fish fry may die because of a direct effect of cercariae irrespective of whether they are exposed to the cercariae of a fish-specific or a non-fish-specific trematode. As regards the further development of larval flukes, this pathological form of colonisation is a dead-end street anyway, as from the dead fish the larvae have a slim chance of getting into the next host. Our experiments demonstrate the following three possibilities of this host-parasite relationship interrupting the developmental cycle:

(1) During the normal life cycle, the infective metacercariae of Diplostomum species appear in the eye only several days after the invasion (Yamaguti, 1975). Therefore, even if an aquatic bird ate the fish fry that died in the fish pond within a few hours of infection - which is rather unlikely because of the small size of the fish -, the fluke would not develop further in it.

(2) In their natural habitat, the xiphidiocercariae used in the experiment wriggle themselves either directly into a tadpole or into another snail (Yamaguti, 1975). Dead fish fry will not serve as food for frogs that look for a moving prey; thus the xiphidiocercariae killing the fish cannot develop further in this way.

(3) Like other authors (Yamaguti, 1975; Evans et al., 1981), we have also observed that in most cases the emerged echinocercariae again get into snails under natural conditions. On lakes, aquatic birds become infected by consuming the snails that already contain metacercariae; therefore, rapidly decomposing dead fish fry sinking to the bottom would have a low chance of transmitting these flukes to their subsequent host.

The cause of death of the experimental fish could easily be inferred from the mode of cercarial penetration and the symptoms preceding death. In the case of Diplostomum cercariae penetrating into the fish in all parts of the body both percutaneously and per os it is clear that abnormal movements and death are caused by the larvae migrating into the central nervous system. The rapid invasion of these cercariae is not accompanied by signs of oxygen deficiency and the pathological process is of rapid course. There is no time for the development of inflammatory processes either, as the larvae do not stay at one place. The aggressive and targeted migration of cercariae towards the head (RatanaratBrockelman, 1968) and their exceedingly large amount as compared to the host's size will invariably lead to the death of the fish. 
Xiphidiocercariae thoroughly penetrate the body of the fish and obviously affect the functioning of all the organs they attack. Death occurs as a result of the gross trauma and dysfunction of the organs. At the same time, these larvae do not get into the fish in as large numbers as do Diplostomum larvae, and some fish may escape massive invasion. One of the possible reasons may be that only the xiphidiocercariae moving near the water surface are really infective (Styczynska-Jurewicz, 1962; cit. by Ginetsinskaya, 1988), and certain fish came into contact primarily with such larvae.

We found that echinocercariae, which entrench themselves in the gill cavity only, are likely to cause injury to the fish through their mechanical effect. The thin-walled cercarial capsules loosely adhering to the wall of the gill cavity may have become detached in some cases, and possibly we did not find all cercariae in the histological sections. The only thing that we could determine with complete certainty by histological methods was that the only extraintestinal location that these cercariae reached was the gill cavity.

As indicated by the histological findings, these latter cercariae do not destroy the respiratory epithelium; rather, because of their substantial size as compared to that of the fish fry, they may mechanically obstruct the flow of water. They may also prevent normal closure of the operculum, which is essential for normal gill function. In any case, these cercariae killed the fish by causing oxygen deficiency, which was indicated also by the gasping of fish before their death.

The amount of larval flukes to be used for infecting the fish was determined roughly from the amount of cercariae that emerge from a snail at a time in the case of the given trematode species. Taking this into consideration, the experiments indicate that under fish pond conditions, where snails with varying degrees of infection occur together, a lower number of snails carrying Diplostomum cercariae or xiphidiocercariae may be sufficient to kill a given number of fish than what is needed in the case of echinocercariae. However, we cannot judge the degree of natural pathogenicity as we do not know the actual density at which the emerged cercariae occur in the ponds, nor do we know the actual frequency of fish exposure to the cercariae. Based upon these experiments it is impossible to determine the precise length of time that the invading cercariae required to kill the individual fish fry, as new cercariae could emerge from the snails throughout the experiment, i.e. not all cercariae were present in the water at the outset. Echinocercariae probably get into the gill cavity accidentally rather than by target-oriented movement, during the respiratory movements of the fish or when they are being eaten by the fish as planktonic food. According to Ginetsinskaya (1988), most cercariae - including even those of Diplostomum come into contact with the host accidentally rather than as a result of an active search for a host. This seems to be supported also by the results of Styczynska- 
Jurewicz (1958) who found that the distribution and movement of cercariae present in the water of ponds were independent of the distribution of fish hosts.

It is a known phenomenon in the fish propagation practice that in many cases the whole population of young fry placed out for the first time is lost, while fry hatched in the same way and placed into the same pond some weeks later will survive. A possible explanation of this phenomenon may be that individuals of the first stock placed into the pond collect into their body the cercariae that develop by the spring and thus remove them from the pond, while older snails which became infected in the previous year and harbour mature cercariae die. Since cercariae ripe for emergence cannot develop in 1-2 weeks in snails that contracted infection from the definitive hosts in the spring, and as most of the trematode cercariae of snails infected earlier have already been shed, the second stock placed out will not be exposed to a cercarial invasion as massive as that affecting the first one. This second stock will thus survive the dangerous period of life, and subsequent cercarial infections will no longer be lethal to it. Field experiments will be necessary to determine whether or not the observed mortality was indeed due to this phenomenon.

Fish may be exposed to cercariae from early spring to late autumn, i.e. throughout the feeding period (Chubb, 1979). If these host-parasite encounters can so easily lead to a biological dead-end street, nothing contradicts the possibility that such unnatural infection processes may occur even in older fish, because of the not too specific host-recognising ability of cercariae and/or their consumption by the fish.

\section{References}

Ballabeni, P. (1994): Experimental differences in mortality patterns between European minnows, Phoxinus phoxinus, infected with sympatric or allopatric trematodes, Diplostomum phoxini. J. Fish Biol. 45, 257-267.

Bauer, O. N., Vladimirov, V. L. and Mindel, N. V. (1964): New knowledge about the biology of Strigeata causing mass diseases of fishes. In: Ergens, R. and Rysavy, B. (eds) Parasitic Worms and Aquatic Conditions. Proceedings of a Symposium held in Prague, Publ. House of the Czechoslovak Academy of Sciences, 1964, pp. 77-82.

Chubb, J. C. (1979): Seasonal occurrence of helminths in freshwater fishes. Part II. Trematoda. Adv. Parasitol. 2, 79-98.

Dobó, Z. (1994): A new fish parasite in our waters: the glochidia of the Amur mussel (in Hungarian). Halászat 87, 62-63.

Dubois, G. (1970): Synopsis des Strigeidae et des Diplostomidae (Trematoda). Mém. Soc. neuchatel. Sci. nat. 10, 256-727.

Edelényi, B. (1974): Trematodes II. Digenea. In: Fauna Hungariae II/5, 117 (in Hungarian). Akadémiai Kiadó, Budapest. 343 pp.

Evans, N. A., Whitefield, P. J. and Dobson, A. P. (1981): Parasite utilization of a host community: the distribution and occurrence of metacercarial cysts of Echinoparyphium recurvatum (Digenea: Echinostomatidae) in seven species of molluscs at Harting Pond, Sussex. Parasitology 83, 1-12. 
Ginetsinskaya, T. A. (1988): Trematodes, their life cycles, biology and evolution. Amerind Publ. Co., New Delhi. 196 pp.

Hoffmann, R. W., Körting, W., Fischer-Scherl, T. and Schaufer, W. (1990): An outbreak of Bucephalus polymorphus in fish of the Main river. Angew. Parasitol. 31, 95-99.

Inchausty, V. H., Foutz, M., Heckmann, R. A., Ruas, C. and Ruas, P. (1997): Diplostomiasis in native and introduced fishes from Yellowstone lake, Wyoming. Great Basin Naturalist 57, 178-183.

Ishkov, M. P. and Batchinskii, V. P. (1969): Diplostomosis of the grasscarp and silver carp in pond farms of Ukraine (in Russian). Mater. nauch. Konf. vses. Obsch. Gelmint. Part I, 101-105.

Kocylowski, B. and Miaczynski, A. (1963): Diseases of Fish (Hungarian translation from Polish). Mezőgazdasági Kiadó, Budapest. 256 pp.

Majoros, G. (1996): Larval trematodes in snails of fish farms (in Hungarian). XXth Fisheries Scientific Meeting. HAKI, Szarvas, Halászatfejlesztés 19, 50-66.

Majoros, G. (1997): Infection of fish fry with cercariae (in Hungarian). XXIst Fisheries Scientific Meeting. HAKI, Szarvas, May 28-29, Abstract no. 40.

Molnár, K. (1974): On diplostomosis of grasscarp fry. Acta Vet. Acad. Sci. Hung. 24, 63-71.

Niewiadomska, K., Valtonen, E. T. and Siddall, R. (1997): Cercariae from Lymnaea stagnalis in lake Kuuhankavesi (central Finland). Acta Parasitol. 43, 132-137.

Ratanarat-Brockelman, Ch. (1968): Migration of Diplostomum spathaceum (Trematoda) in the fish intermediate host. Z. Parasitenkd. 43, 123-134.

Roberts, R. J. (1989): Fish Pathology. Baillière Tindall, London. 265 pp.

Schell, S. C. (1970): How to know the trematodes? W. M. C. Brown Comp. Publishers, Dubuque, Iowa. $355 \mathrm{pp}$.

Schell, S. C. (1985): Handbook of Trematodes of North America North of Mexico. University Press of Idaho, Moscow, Idaho. 263 pp.

Sommerville, C. (1982): The pathology of Haplorchis pumilio (Looss, 1896) infection in cultured tilapias. J. Fish Dis. 5, 243-250.

Styczynska-Jurewicz, E. (1958): Field experiments on the invasion process with the cercaria of Diplostomum spathaceum Rud. in fishes. Wiadomości Parazytologiczne 4, 639-641.

Vojtek, J. (1981): Trematode Fauna of Birds of the Czechoslovak Republic (in Czech). Univerzita J. E. Purkyne, Brno. 212 pp.

Yamaguti, S. (1975): A synoptical review of life histories of digenetic trematodes of vertebrates. Kegaku Publ. Comp. Ltd., Tokyo. 590 pp.

Zdárská, Z. (1963): Larval trematodes of freshwater snails in Czechoslovakia (in Slovak). Československa parasitologie 10, 207-262. 
